\title{
Mulmiges Gefühl beim Einsatz von Plazebos
}

Djalali, S ; Senn, O

\begin{abstract}
Plazebos sind allgegenwärtig - nicht nur als Vergleichsgruppe im Rahmen kontrollierter Studien, auch in der täglichen Praxis werden sie häufig eingesetzt. Das belegen bereits zahlreiche Studien. Doch jetzt wurde erstmals untersucht, was genau Hausärzte vom Plazeboeffekt überhaupt erwarten.
\end{abstract}

Posted at the Zurich Open Repository and Archive, University of Zurich ZORA URL: https://doi.org/10.5167/uzh-79789

Journal Article

Published Version

Originally published at:

Djalali, S; Senn, O (2013). Mulmiges Gefühl beim Einsatz von Plazebos. PrimaryCare, 13(5):89. 


\title{
Mulmiges Gefühl beim Einsatz von Plazebos
}

\author{
Bericht über eine Studie aus dem Institut für Hausarztmedizin, Universität Zürich [1]
}

\author{
Plazebos sind allgegenwärtig - nicht nur als Ver- \\ gleichsgruppe im Rahmen kontrollierter Studien, auch \\ in der täglichen Praxis werden sie häufig eingesetzt. \\ Das belegen bereits zahlreiche Studien. Doch jetzt \\ wurde erstmals untersucht, was genau Hausärzte vom \\ Plazeboeffekt überhaupt erwarten.
}

Plazebo ist nicht gleich Plazebo. Tatsächlich gibt es einen kleinen, aber durchaus relevanten Unterschied zwischen reinen und unreinen Plazebos. Aber sind sich Mediziner, die Plazebos einsetzen, dessen bewusst und setzen sie die Plazebosorten gezielt ein? Mit semistrukturierten Interviews wurde erstmals die Einstellung und Erwartung von Hausärzten gegenüber Plazebos untersucht.

\section{Rein oder unrein, das ist hier die Frage}

Reine Plazebos sind Substanzen, die keinerlei pharmakologischen Effekt haben, etwa reine Glukosepillen. Dagegen handelt es sich bei unreinen Plazebos um eine pharmakologisch wirksame Substanz, deren Wirkung sich jedoch nicht auf das zu behandelnde Krankheitsbild entfaltet. Ein Beispiel wären Antibiotika oder Vitamine, die bei einem viralen Infekt verabreicht werden.

\section{Hausärzte im Interview}

12 Schweizer Hausärzte nahmen an der Interviewstudie teil und wurden unter anderem gebeten, ihre Definition von «Plazebo» zu formulieren. Im Ergebnis entsprach die Mehrheit der Antworten der Definition eines reinen Plazebos. Konfrontiert mit Beispielen für unreine Plazebos, gab nur eine Minderheit an, diese ebenfalls als Plazebos anzusehen. Die Mehrheit der Ärzte war sich also des Ausmasses der Plazebodefinition und damit des Ausmasses des tatsächlichen Plazebogebrauchs in der Praxisroutine nicht bewusst.

\section{Ein Mittel gegen psychosomatische Beschwerden}

Weiterhin ergab die Befragung, dass Plazebos in der Hausarztpraxis mehrheitlich bei harmlosen Gesundheitsproblemen eingesetzt werden, für die keine zufriedenstellende somatische Erklärung gefunden werden kann - Fälle, in denen häufig auch andere alternativmedizinische und komplementärmedizinische Therapien zum Einsatz kommen. Diesen sprechen die befragten Hausärzte ebenfalls einen gewissen Plazeboeffekt zu.

\section{Ethische Bedenken}

Auch wenn der Plazeboeinsatz nichts Ungewöhnliches in der Hausarztmedizin zu sein scheint, gaben alle Befragten an, dass die ethischen Aspekte solcher Behandlungen unklar seien und dass sie unsicher seien, wie sie die Anwendung von Plazebos ihren Patienten kommunizieren sollten. Die meisten würden offizielle ethische Richtlinien als Unterstützung sehr begrüssen.

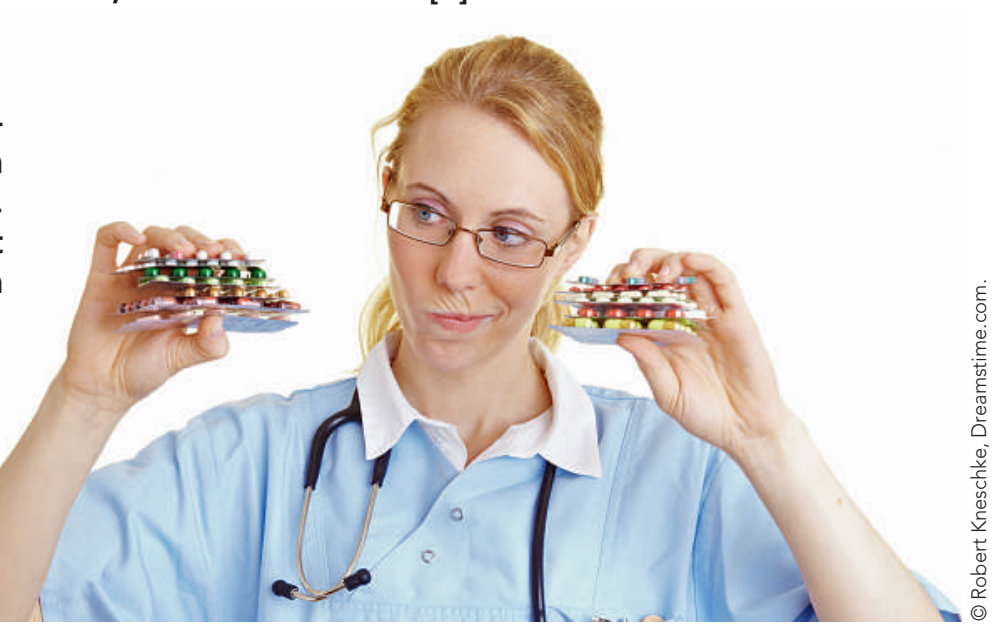

Reines Plazebo oder unreines? Ein feiner Unterschied, der nicht immer klar ist ...

\section{Guidelines beruhigen}

Viele Hausärzte scheinen sich nicht bewusst zu sein, dass manche von ihnen verordneten Medikamente als unreine Plazebos klassifiziert werden. Offizielle Empfehlungen würden von vielen Ärzten in diesem Kontext als wertvolle Hilfe gesehen, um über die Unsicherheiten rund um den Plazeboeinsatz hinwegzuhelfen. Das Thema an sich steht aber für viele Hausärzte in einem gewissen Konflikt mit der Wahrnehmung der eigenen Rolle als professioneller und verantwortungsbewusster Mediziner. Der Unterschied zwischen reinen und unreinen Plazebos wird mehrheitlich nicht gemacht bzw. ausgeblendet.

\section{Referenz}

1 Fent R, Rosemann T, Fässler M, Senn O, Huber CA. The use of pure and impure placebo interventions in primary care - a qualitative approach. BMC Fam Pract. 2011;12:11.

\section{PrimaryResearch - das Fenster zur Forschung} In einer Artikelserie stellen wir die Forschungsarbeiten vor, die das Institut für Hausarztmedizin an der Universität Zürich (IHAMZ) seit seiner Gründung publiziert hat. Die Originalarbeiten sind entweder «open access» zugänglich oder beim jeweiligen Autor auf Anfrage erhältlich. Die Ergebnisse geben einen spannenden Einblick in die täglichen Herausforderungen, aber auch die Leistung der Hausarztmedizin.

An dieser Stelle ein herzlicher Dank an alle Kolleginnen und Kollegen, die sich an den Projekten beteiligen und die nachfolgenden Ergebnisse erst ermöglicht haben!

Ihr IHAMZ Team

\section{Korrespondenz:}

Dr. med. Sima Djalali

Institut für Hausarztmedizin

Universität Zürich

Pestalozzistrasse 24, 8091 Zürich

sima.djalali[at]usz.ch 